\title{
Controlling a Large CPU Farm Using Industrial Tools
}

\author{
Alba Sambade Varela, Markus Frank, Domenico Galli, Clara Gaspar, Beat Jost, Niko Neufeld, and \\ Eric van Herwijnen
}

\begin{abstract}
The LHCb experiment at CERN will have an Event Filter Farm (EFF) composed of 2000 CPUs. These machines will form a pool of 50 sub-farms with 30 to 40 nodes each, running a large amount of High Level Trigger (HLT) tasks in parallel. Although these tasks are identical algorithms, they can run at the same time being configured with different parameters, such as run type (Physics, Cosmics, Test, etc.) or with different subdetectors (partitions). The HLT is the second of the two trigger levels in LHCb. Its selection algorithms reduce the incoming data rate of $1 \mathrm{MHz}$ to an output rate of $2 \mathrm{kHz}$. Selected events are sent for mass storage and subsequent offline reconstruction and analysis. These trigger processes running online are based on the same software framework as the algorithms for offline analysis (Gaudi). The control of the trigger farm was developed with an industrial SCADA system (PVSS) which is used throughout the Experiment Control System (ECS). The HLT algorithms are handled by the ECS like hardware devices, for instance, high voltage channels. The integration of the HLT controls in the overall ECS, which is modeled as finite state machines, will be presented.
\end{abstract}

Index Terms-Experiment control system (ECS), finite state machine (FSM), gaucho, high level trigger (HLT), PVSS.

\section{INTRODUCTION}

$\mathbf{T}$ HE LHCb experiment [1] is designed to exploit the large number of bb-pairs produced in pp interactions at $14 \mathrm{TeV}$ at the $\mathrm{LHC}$, in order to make precise studies of $\mathrm{CP}$ asymmetries and rare decays of b-hadrons. A filter is applied online to this huge amount of events so that only interesting events are written to storage, at a reduced frequency, for further offline analysis. The event reduction is made in two levels:

- A first hardware trigger implemented in custom electronics: L0-trigger. It reduces the rate from $40 \mathrm{MHz}$ to $1 \mathrm{MHz}$.

- High Level Trigger (HLT), a software filter running on an Event Filter Farm (EFF) that reduces the incoming data rate of $1 \mathrm{MHz}$ to an output rate of $2 \mathrm{kHz}$.

The control of the EFF implies both the control of the hardware, a pool of 2000 CPUs, and the control of the software, the trigger processes running throughout the farm. The first one is carried out by the Farm Monitoring and Control System (FMC) application [2], designed to monitor and control each PC and to

Manuscript received June 19, 2009; revised October 03, 2009 and November 25, 2009. Current version published April 14, 2010.

A. Sambade Varela, M. Frank, C. Gaspar, B. Jost, N. Neufeld, and E. van Herwijnen are with the European Organization for Nuclear Research, CERN, CH1211 Switzerland (e-mail: alba.sambade.varela@cern.ch; markus.frank@cern.ch; clara.gaspar@cern.ch; beat.jost@cern.ch; niko. neufeld@cern.ch; eric.van.herwijnen@cern.ch).

D. Galli is with the INFN, Universita di Bologna, Bologna, Italy (e-mail: domenico.galli@bo.infn.it).

Color versions of one or more of the figures in this paper are available online at http://ieeexplore.ieee.org.

Digital Object Identifier 10.1109/TNS.2009.2039354 supervise the overall status of the farm. The second one is implemented with Gaucho [3] (GAUdi Component Helping Online), a package to control and monitor the trigger processes [4]. To guarantee a coherent integration of the Event Filter Farm controls into the global Experiment Control System (ECS), these packages have been implemented with the commercial software used throughout the ECS.

\section{EXPERIMENT CONTROL SYSTEM}

The ECS [5] is in charge of the configuration, operation and supervision of all the online components in LHCb. It is characterized by using the same set of tools for the implementation and by following the same architecture throughout the system to integrate all items to be controlled. The HLT control was consequently developed according to the same design principles followed with any standard device, such as a temperature sensor or a control board.

It has been implemented using a commercial SCADA (Supervisory Control and Data Acquisition) system, PVSS [6], used extensively for the supervision and control of industrial processes. Based on this software, and in common for the four LHC experiments, a framework toolkit has been developed. One principal component used from this framework is the Finite State Machine (FSM) package, based on SMI++ [7]. SMI++ is a toolkit that provides for rule-based automation and error-recovery. It allows implementing a control system with a hierarchical architecture and modeling its behavior in terms of states and actions i.e., of finite state machines; thereby, the LHCb ECS is a tree-like structure of logical nodes. Every component to be controlled, or group of components, is integrated in the system as one logical node. These nodes can be of two types: Control Units (CU) and Device Units (DU).

The implementation of the HLT control hierarchy is shown in Fig. 1, where is also depicted its integration into the $\mathrm{LHCb}$ ECS hierarchy. The root of the HLT tree is a CU that links the HLT to the main ECS node. The hierarchy is further brokendown following first a physical division (subfarms and nodes) and then a logical division (algorithms). The upper layers are made up of CUs which implement the behavior of the system. They can take local decisions and distribute commands. In this architecture, if a command is sent from the HLT control node (or top node ECS), it is propagated down the tree through the whole pool of subfarms until it finally reaches the lowest level. On the other hand, the state of the top nodes summarizes the state of the nodes that are under it. Another capability of the $\mathrm{CU}$ is the partitioning: a node can be excluded (or included) from the system so that there is an independent hierarchy that can run in parallel. The lowest layer consists of DUs which are the interface to real devices. In the HLT case the devices are software processes and, as can be seen in Fig. 1, there is a DU 


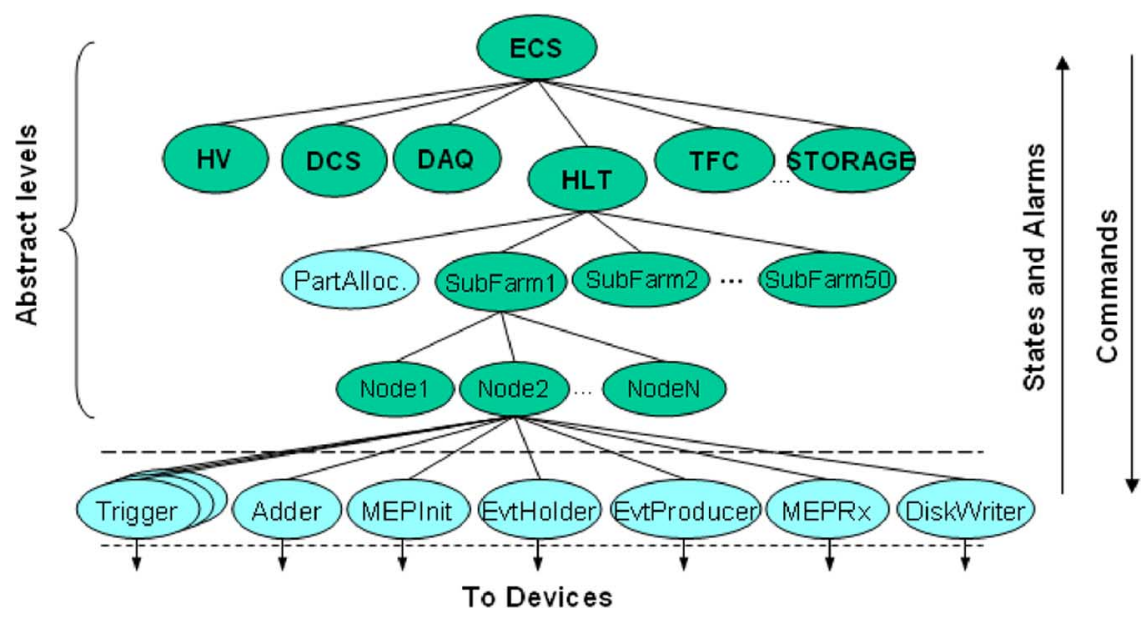

Fig. 1. HLT control hierarchy and its integration into LHCb ECS.

per process. They receive commands from the upper levels and implement them as parameters for the HLT algorithms to have a real action executed.

The whole HLT hierarchy runs in different control projects (PVSS projects) spread out over a network of control PCs. There is a main HLT control project which runs in a dedicated control PC and the pool of HLT CPUs (grouped into 50 subfarms, 50 racks, with up to 40 nodes each) is controlled by 50 independent control PCs running each a control project. Each project corresponds to the controls of a single subfarm and runs an independent FSM hierarchy. All the subfarm hierarchies are integrated in parallel as subpart of the LHCb ECS tree linked through the HLT project, as can be seen in Fig. 1. This fact, together with the concept of partitioning (the possibility to exclude sub-trees in a hierarchy), allows to integrate new farms in the hierarchy at any time. Several procedures have been implemented to automate the management of the control pool, being able to recreate the 50 subfarm control projects in $\sim 300 \mathrm{sec}-$ onds. In addition, all HLT control projects share the framework toolkit installation. This avoids redundancy and problems of versioning, therefore reducing the maintenance needed.

\section{High LeVEL TRIGGer Node}

\section{A. Task Architecture on HLT Node}

Due to the complex nature of the selection of B-events, which are the main interest of LHCb, it is required that the HLT algorithms have access to the complete data of an event. This means that the fragments belonging to the same original collision (spread out over all the Readout Boards) have to be collected in the same place [8]. A first task, MEPRx, makes sure that all MEP ${ }^{1}$ from the same events are received. Then multi events are assembled by an Event Builder (Event Holder and Event Producer) [9] and data is further distributed to event filter processes (selection or trigger algorithms). There is one selection algorithm running per core (i.e., 8 algorithms per HLT node, in the current processors). They compute trigger decisions and forward the accepted events to the data writer task, DiskWriter,

\footnotetext{
${ }^{1}$ Multi Event Packet: event fragments grouped together to minimize the over-
} head on the network.

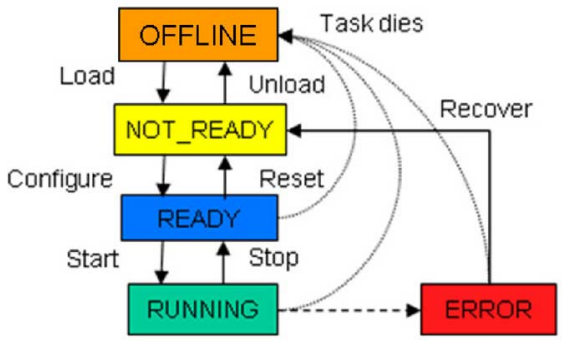

Fig. 2. FSM states and transitions of a HLT process.

which sends these data to the storage system. Also monitoring tasks run on the EFF to study the HLT rejected events, which are not available later, while a small part of the HLT accepted events is also forwarded to dedicated farms for calibration and monitoring,

All the trigger algorithms are implemented using Gaudi [10], an experiment-independent software framework for event data processing applications. The fact that the same software is used both for the HLT algorithms in the EFF (running online, that is at run time) and for the offline physics analysis, brings some homogeneity and smooths out the boundaries between online and offline.

\section{B. Control of Trigger Processes}

While the HLT algorithms are specific software processes, the framework used to control and integrate them into the ECS is the same used throughout the experiment to control any kind of device, whether custom or standard. Thus every HLT process is integrated into the ECS and controlled by a DU, whose behavior is implemented as Finite State Machines. The state machine diagram, which can be seen in Fig. 2, is common for all the processes running on a HLT node.

As mentioned before, these DU are located at the bottom of the control hierarchy and they receive commands from the subfarm node $\mathrm{CU}$ above them (Fig. 1). Thus, a state change can be triggered either by sending a command or as consequence of a change in monitored task parameters. This last one is the case of the automatic transitions represented with dashed lines in Fig. 2. The FSM transitions are mapped to Gaudi transitions. Whenever 


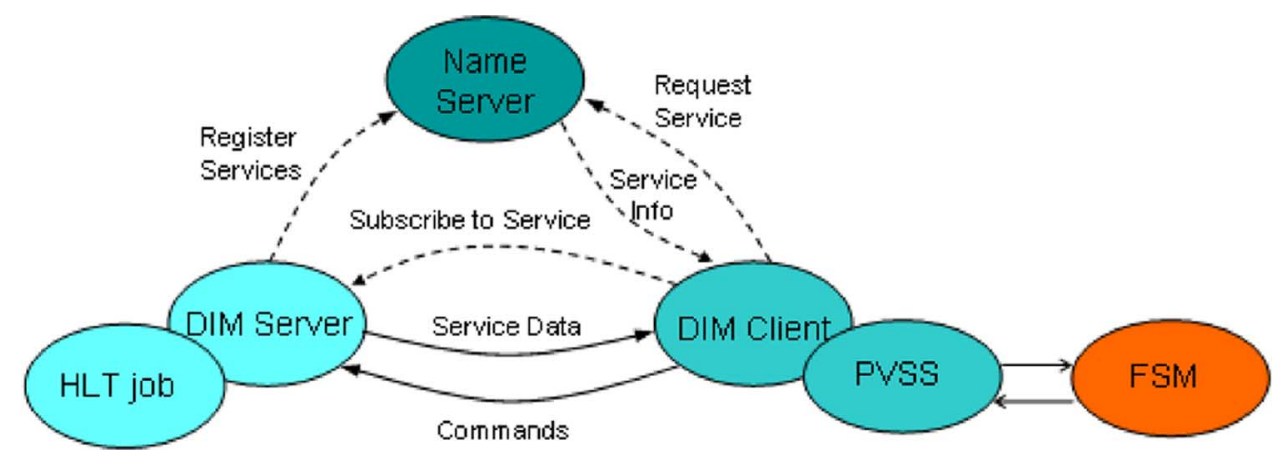

Fig. 3. DIM communication mechanism in the HLT context.

the process DU receives a command, it executes a script associated to it and the command is translated into an action done in the HLT process. The other way around, if a HLT algorithm dies for whatever reason, its corresponding DU will transit to the OFFLINE FMS state, which will trigger the subfarm node $\mathrm{CU}$ going to OFFLINE state, and so on higher in the hierarchy.

Even if a command can be sent in parallel to the different DU belonging to a $\mathrm{CU}$, the different algorithms running on the HLT farm need to be loaded, configured and started in a specific order. The farm node CU implements this automatic specific sequencing of actions.

Depending on the objectives of a run, it is desired to configure the HLT processes with different settings. (In general, this is extended to every resource in the ECS). The desired running mode (PHYSICS, CALIBRATION, COSMICS. ..), is specified by the parameter "RUN_TYPE" which is sent with the command "Configure". These configuration settings are applied at run time.

Communication Layer: To translate the HLT task state into a FSM state, and to send commands from the FSM object to the HLT process, a communications mechanism has to be established. The Distributed Information Management system (DIM) [11], plays this key role in the HLT control. DIM is a network communication layer built on top of the TCP protocol and based on the client-server paradigm. The basic concept in the DIM approach is the concept of service. A service is normally a set of data (of any type or size) and it is recognized by a name ('named services'). The server provides data services and command services to clients by registering them (normally once, at startup) with the DIM Name Server, DNS. The client subscribes to services by asking the DNS which server provides the service and then contacting the server directly. The HLT task is a DIM server. The HLT process publishes their information (status of the task itself, counters, messages, alarm messages and histograms) which is collected by a PVSS DIM client application and stored in datapoints (variables of the process image in a PVSS system). The FSM object (DU) is connected to these datapoints and will react when its value changes; the DU state will be recalculated (changing or not) and also actions in parallel can be triggered. This relation can be seen in Fig. 3. A similar procedure takes place on the reverse direction for the transmission of commands: the DU writes to datapoints that trigger the DIM client to send the command to the DIM server (HLT process).
While the name space for services is free, the control of the EFF is based on a HLT naming convention: HLT processes are identified by a unique string called the UTGID and the subfarms and nodes by a row and index suffix.

\section{PARTITIONING}

LHCb is composed of several subdetectors collecting data together. While this is the normal mode of operation for physics, each subdetector can be run by itself, the so called stand-alone mode. This is mostly the case during commissioning stage, but will be also the case between fills when some calibration or tests need to be performed by experts. Because of this, the $\mathrm{LHCb}$ DAQ system has the concept of Partition, which defines which part of the whole detector is used [12]. The detector is divided in 16 parts, so there is the possibility of independent, parallel running parts of a sub-detector, an entire sub-detector or a combination of sub-detectors. To run each partition, it needs a set of the common resources, i.e., a part of the Timing and Fast Control, a part of the High Level Trigger Farm, a part of the Storage and a part of the Monitoring system. In this scenario, the first step for starting a run is the allocation of these common resources. When sending the "Allocate" command, the system will reserve one Readout Supervisor module, a number of subfarms, a storage slice (file writing tasks) and a monitoring slice (monitoring tasks). This reservation procedure is done dynamically and is implemented by several specific Device Units, the Allocators. The Farm Allocator (PartAlloc), whose integration in the HLT control system is shown in Fig. 1, receives the command from the ECS top node through the HLT CU and checks which farms are free and assigns them to the specific partition.

\section{GRAPHICAL USER INTERFACE}

The LHCb experiment will be run by non-expert shifters in the Control Room. This fact brings the need of reducing the space for the operator intervention. To achieve this, the $\mathrm{LHCb}$ ECS was implemented in a way that complete automation can be reached. This is thanks to the FSM package. Additionally, the system will be entirely operated through a graphical user interface. The GUI implemented is a user friendly, intuitive for the shifter, set of PVSS panels, from where the HLT farm (subfarms and HLT processes) can be fully operated (configured, started, stopped, etc.). Likewise, the information published by the HLT tasks (counters, rates) is summarized and displayed on 


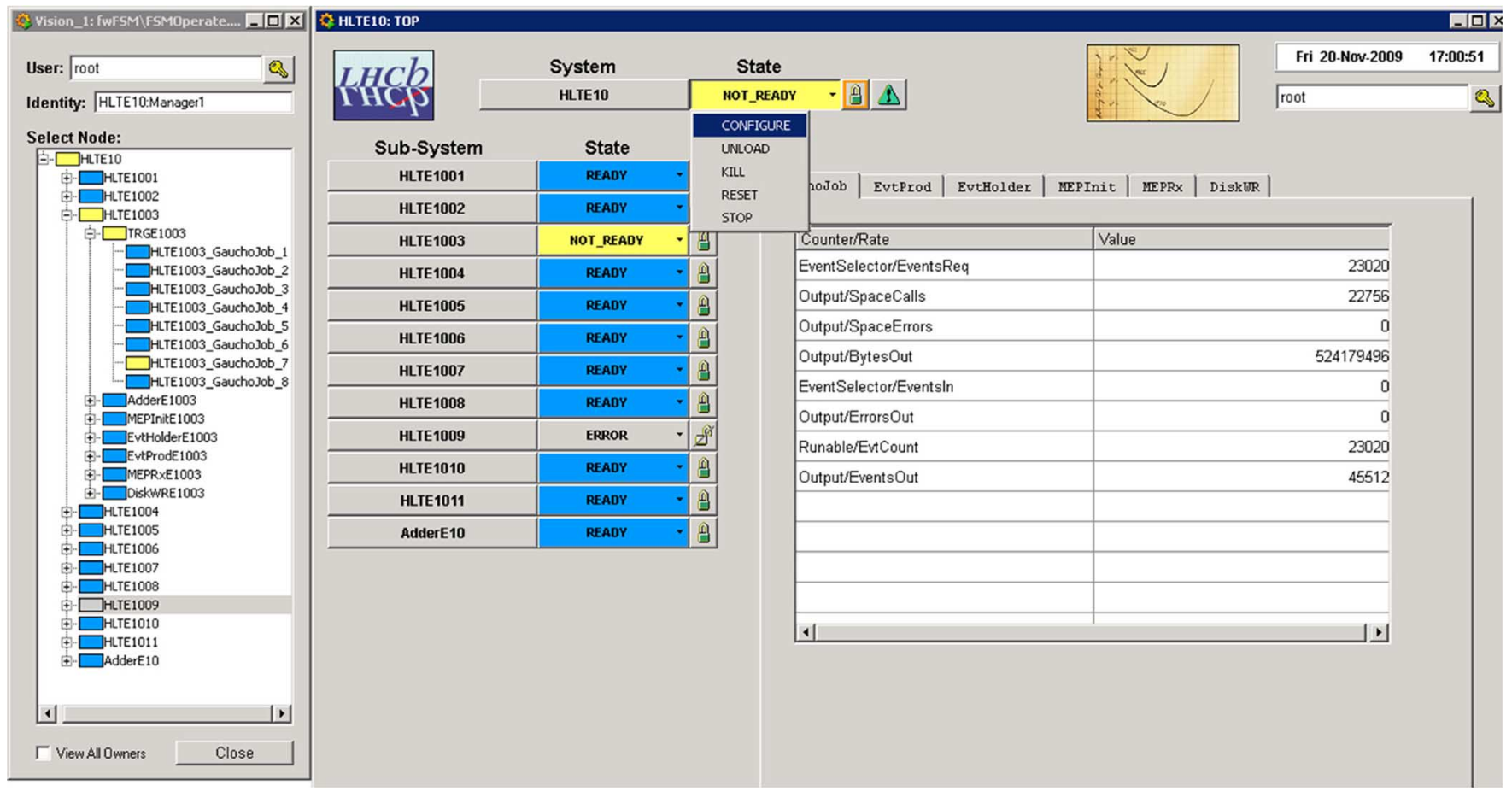

Fig. 4. HLT subfarm hierarchy and control panel.

the panels. Fig. 4 shows the HLT GUI controls for a subfarm. On the left, there is the tree hierarchy and on the right its corresponding control panel.

The shift operator can navigate down through the hierarchy to find out detailed information about a node in particular.

\section{STATUS}

Nowadays, the HLT pool is composed of 550 CPUs, having operational the 50 subfarms with 11 nodes each.

It turned out that the time of configuring the whole farm was longer than expected. It takes $\sim 120$ seconds to configure (most of the time taken by the trigger processes) and some work to reduce this time is ongoing in this field.

At early stage of the implementation some communication loss between the HLT processes and the control project was observed. To solved this and assure the control over the processes at any time, recovery procedures and timeouts were implemented.

\section{CONCLUSION}

The control system for the LHCb High Level Trigger farm has been described in this paper. The control and supervision of the Gaudi trigger processes was implemented with the same set of tools that is used to control all the online resources; namely, a commercial SCADA software, PVSS, customized by a CERNwide common framework. The HLT control hierarchy was integrated in the homogeneous LHCb Experiment Control System, in charge of the configuration, operation and monitoring of the whole experiment. The implemented HLT control system facilitates the steering of a single subfarm, or a subset of them, by sending commands, in a "single-click" base, and allows the operation by non-expert users. The HLT control system has now reached a production-quality level and actually constitutes a fundamental building block of the LHCb online system.

\section{REFERENCES}

[1] LHCb collaboration, "LHCb-the Large Hadron Collider Beauty Experiment, Reoptimised Detector Design and Performance 2003," CERN/ LHCC 2003-030.

[2] D. Galli et al., "The monitoring and control system of the LHCb event filter farm," IEEE Trans. Nucl. Sci., vol. 55, no. 1, Feb. 2008.

[3] E. van Herwijnen, "Control and monitoring of on-line trigger algorithms using Gaucho," presented at the ICALEPCS, Geneva, Switzerland, 2005, WE3A.5-60.

[4] P. Vannerem, C. Gaspar, B. Jost, and N. Neufeld, "Distributed control and monitoring of high-level trigger processes on the LHCb on-line farm," presented at the ICALEPCS, Gyeongju, Korea, 2003.

[5] C. Gaspar et al., "An integrated experiment control system, architecture, and benefits: The LHCb approach," IEEE Trans. Nucl. Sci., vol. 51, no. 3, Jun. 2004.

[6] PVSS [Online]. Available: http://www.pvss.com

[7] B. Franek and C. Gaspar, "SMI++, object oriented framework for designing and implementing distributed control systems," IEEE Trans. Nucl. Sci., vol. 47, no. 2, Apr. 2000.

[8] N. Neufeld et al., "LHCb online event processing and filtering," $J$. Phys.: Conf. Ser. 119 (2008), CHEP, 022003.

[9] M. Frank et al., "The LHCb high level trigger infrastructure," J. Phys.: Conf. Ser. 119 (2008), CHEP, 022023.

[10] G. Barrand et al., "GAUDI-A software architecture and framework for building HEP data processing applications," presented at the CHEP, Padua, Italy, 2000.

[11] C. Gaspar, M. Donzelmann, and Ph. Charpentier, "DIM, a portable, light weight package for information publishing, data transfer and inter-process communication," Computer Physics Communications. Padua, Italy, 2000, CHEP.

[12] O. Callot, "Running LHCb" course, LHCb shift training [Online]. Available: https://lbtwiki.cern.ch/pub/Operation/TrainingSlides/Running_LHCb_v1r1.pdf 